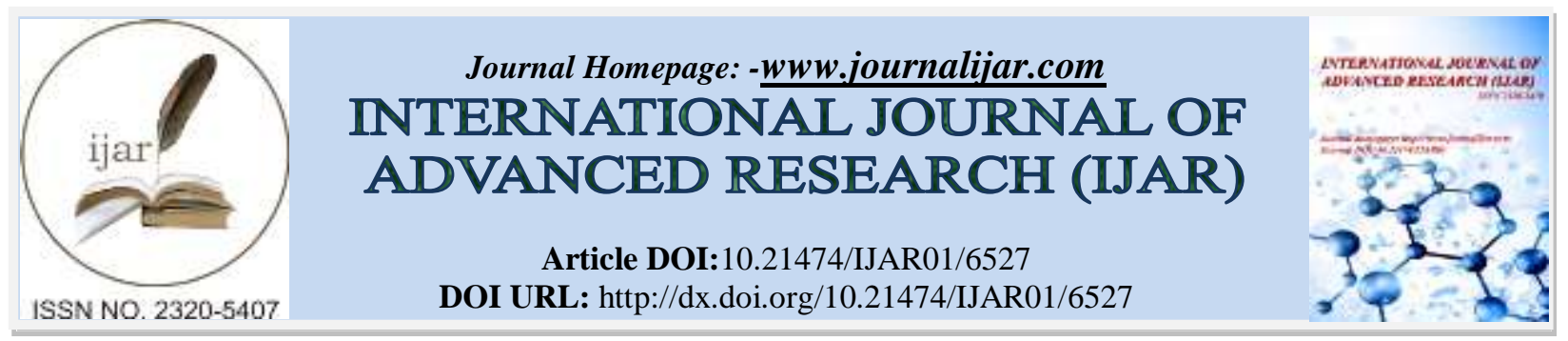

RESEARCH ARTICLE

\title{
KEEP ME ALIVE: REVASCULARIZATION IN IMMATURE PERMANENT MOLAR.
}

\section{Dr. Pooja Patel ${ }^{1}$, Dr. Shoba Fernandes ${ }^{2}$, Dr. Jayasudha ${ }^{3}$, Dr. Yash Bafna ${ }^{4}$, Dr. Kuldip Shah ${ }^{5}$, Dr. Mayur Vaghasiya $^{6}$ and Dr. Jimisha Patel ${ }^{6}$.}

1. Post Graduate Student, Department of Pediatric and Preventive Dentistry, Narsinhbhai Patel Dental College and Hospital, Visnagar, India.

2. Professor and Head, Department of Pediatric and Preventive Dentistry, Narsinhbhai Patel Dental College and Hospital, Visnagar, India.

3. Professor, Department of Pediatric and Preventive Dentistry, Narsinhbhai Patel Dental College and Hospital, Visnagar, India.

4. Reader, Department of Pediatric and Preventive Dentistry, NPDCH, Narsinhbhai Patel Dental College and Hospital, Visnagar, India.

5. Senior lecturer, Department of Pediatric and Preventive Dentistry, Narsinhbhai Patel Dental College and Hospital, Visnagar, India.

6. Post Graduate Student, Department of Pediatric and Preventive Dentistry, Narsinhbhai Patel Dental College and Hospital, Visnagar, India.

\section{Manuscript Info}

(.........................

Manuscript History

Received: 13 December 2017

Final Accepted: 15 January 2018

Published: February 2018

Keywords:-

Sponge, Levantine, Mediterranean, Antibacterial Agent, Pathogenic Bacteria.

\section{Abstract}

Necrotic immature teeth present a challenge to dentists because the techniques used in apexification leave the tooth susceptible to fracture, since the root does not continue to grow in length and the canal walls are thin. Revascularization has emerged as an alternative to resolve these deficiencies, enabling apical closure, continued development of the roots, and thickening of the dentinal walls. Case report: clinically and radiographically diagnosed necrotic immature permanent tooth was treated via revascularization treatment. The therapeutic protocol involved accessing the pulp chamber; irrigating copiously with saline; applying a double antibiotic paste as intracanal dressing; then provisionally sealing it. After 3 weeks, the canal was cleaned and the apex irritated with a size $15 \mathrm{~K}$-file to induce blood that would serve as a scaffold for pulp revascularization. MTA was used as a coronal plug. Follow up of 3, 6 and 9 months presented.

Copy Right, IJAR, 2018,. All rights reserved.

\section{Introduction:-}

Treatment of necrotic immature teeth is a challenge due to the thin dentinal walls and short roots which make them more susceptible to fracture. The treatment of choice is apexification, which consists of inducing apical closure by using Calciumhydroxide or Mineral Trioxide Aggregate (MTA). ${ }^{1,2}$ However, while apexification resolves the problem of apical periodontitis and induces apical closure, enabling definitive root canal therapy to be performed, it does not thicken the root walls or allow the root to continue to develop, leaving the tooth susceptible to fracture. ${ }^{3}$ Revascularization is a new treatment method as a part of tissue engineering for immature necrotic 
permanent teeth. Tissue engineering is a growing field. In the near future, it will probably be possible to generate a complete vital tooth from a single stem cell. Pulp revascularization is dependent on the ability of residual pulp and apical and periodontal stem cells to differentiate. ${ }^{4,5,6}$ These cells have the ability to generate a highly vascularized and a conjunctive rich living tissue and is able to colonize the available pulp space. The protocols referred to as regenerative endodontic procedures are currently being revived as a new paradigm in conservative treatment. It was previously thought that regenerating the necrotic pulp of an immature tooth with periapical periodontitis was impossible; nowadays however we know that, in a suitable medium, this can be achieved using the technique known variously as "revascularization" "7,8 or "revitalization". ${ }^{9}$ For the revascularization proper disinfection of the canal, a suitable matrix for new tissue in-growth, and an effective seal for the coronal access are vital for a satisfactory result, ${ }^{10}$ equally important is a supply of dental stem cells so that revascularization can be successfully completed. ${ }^{11}$ This case presents a successfully treated single tooth with 9 months follow up.

\section{Case Report:-}

A 9-year-old patient presented with symptoms in lower left posterior region suggesting acute pulpitis, with a sharp pain that increased at night and did not ease with analgesics. No abscess was observed upon clinical examination, although there was a large cavity. On periodontal examination, no pathologic tooth mobility was observed, and probing depth was less than $3 \mathrm{~mm}$. Radiographically, caries extending into the pulp chamber of 36 was observed. The image showed periapical radiotranslucency and open apices. The patient was prescribed preliminary antibiotic treatment (Amoxicillin $50 \mathrm{mg} / \mathrm{kg}$ ). Treatment plan that we arrived at was revascularization. Under local anesthesia and rubber dam, the pulp chamber was accessed (figure 1) and the canal irrigated with $2.5 \% \mathrm{NaOCl}$. Working length was determined with size 10 no K-files (figure 2), minimal instrumentation and constant, copious irrigation. The double antibiotic paste (Metronidazole and Ciprofloxacin) was placed in the canal mixed equal proportions, with saline, and the chamber was temporarily sealed.(figure 3) After 3 weeks, the paste was removed with copious irrigation of saline and $2.5 \%$ Sodium Hypochlorite. A file was inserted into the root canal to induce apical bleeding, allowing 15 minutes for the clot to form. 2mm of MTA was placed in the coronal part of the canal (figure 4) followed by semipermanent restoration. At 3, 6, 9 months radiographic follow up, a decrease in the periapical pathology and increased dentinal wall thickness and partially closed apical foramen was observed. Follow up is continued.

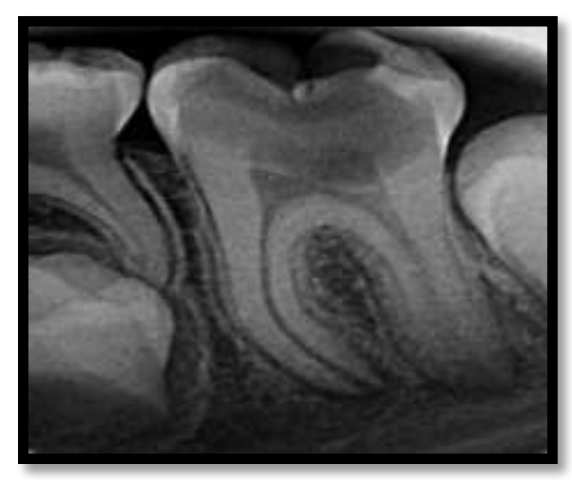

Figure 1:-Pulp chamber was accessed

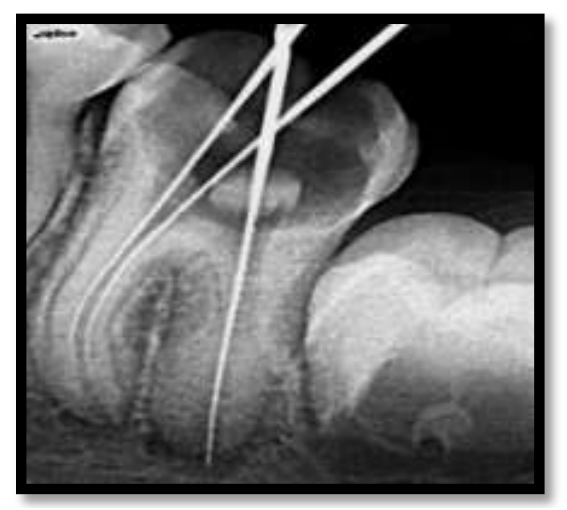

Figure 2:-Working length was measured 

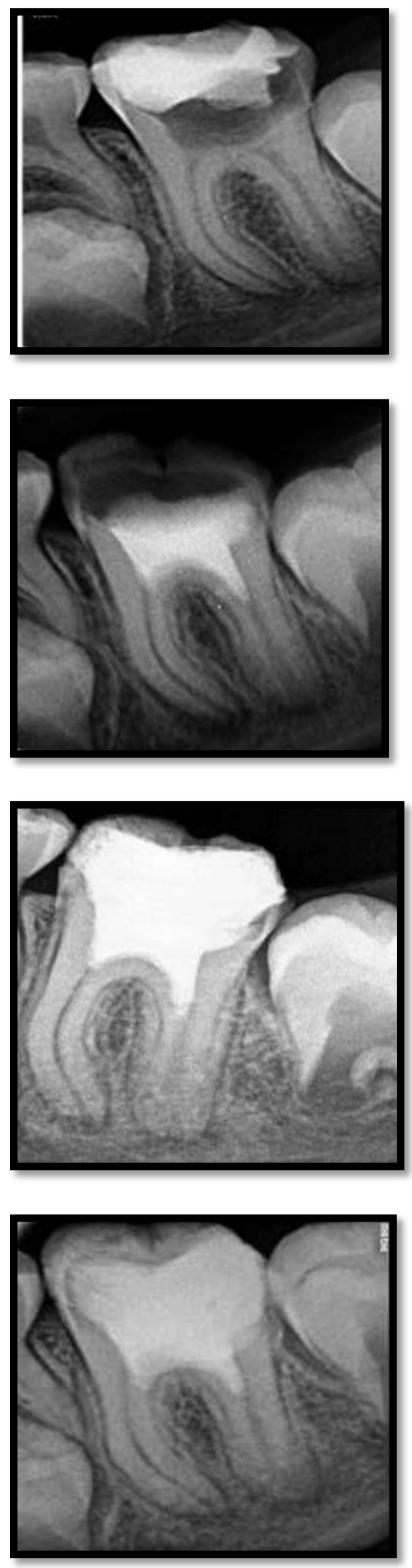

Figure 3:-Intracanal medicament (Ciprofloxacin and Metronidazole) was placed

Figure 4:- After bleeding induced coronal MTA Plug was placed

Figure 5:- 3 months follow up

Figure 6:- 6 months follow up 


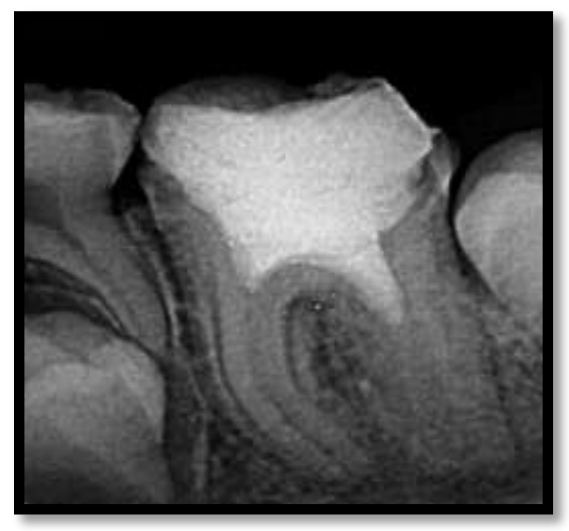

Figure 7: 9 months follow up

\section{Discussion:-}

Pulp regeneration may be defined as the replacement of damaged tissue by cells identical to the lost tissue, leading to the complete reestablishment of biological function.

Revascularization may be defined as the invagination of undifferentiated periodontal cells from the apical region in immature teeth.

The procedure to be followed for revascularization treatment in necrotic immature permanent teeth is not standardized and there is considerable controversy to date. ${ }^{12}$ Nonetheless, substantial numbers of authors seem to support the following protocol. Initial preoperative radiographic evaluation should be carried out to determine the degree of root development and the periapical status of the tooth. Access cavity prepared, followed by antimicrobial irrigants and double or triple antibiotic paste followed by bleeding induced, and placement of MTA plug.

The recommended steps for revascularization in molars were followed in this case. Irrigants play a role of primary disinfection. They should have a maximal bactericidal and bacteriostatic effect and minimal cytotoxic effect on stem cells and fibroblasts to allow their survival and ability to proliferate. In this case Sodium Hypochlorite, saline were the irrigant solutions used. Sodium Hypochlorite remains irrigant of reference in endodontics. It has a solvent action on necrotic tissue and an antiseptic effect widely demonstrated. ${ }^{14}$ Recommended concentrations vary between $0.5 \%$ and $5.25 \%$. Cytotoxicity of Sodium Hypochlorite is proportional to its concentration. The concentration of $2.5 \%$ seems to be the best compromise between efficiency and lack of toxicity. ${ }^{15,16,17}$

Minimal instrumentation was used in the root canals to ensure that the infected pulp tissue was eliminated but without weakening the canals. In accordance with the research of Jadhav et al. ${ }^{18}$ and Chen et al. ${ }^{9}$ involving humans, as well as others who used an animal model, we decided to use gentle instrumentation for the reason mentioned above.

The intracanal medication should be used at concentrations that are bactericidal but do not simultaneously prejudice stem cell viability ${ }^{10}$. The literature describes the use of $\mathrm{Ca}(\mathrm{OH})_{2}$ or a paste consisting of double or triple antibiotics 3,9 .

Bose et al. observed that although the different medications produced apical closure and increased root length, a greater increase in dentinal wall thickness was observed with the triple antibiotic paste. With respect to the triple antibiotic paste, most authors advocate using equal proportions of Ciprofloxacin, Metronidazole, and Minocycline, mixed with sterile water/glycerine/glycol, since the flora in the root canal system is polymicrobial ${ }^{3}$.

A mixture of these three antibiotics has shown satisfactory results for disinfecting the canals and the healing of periapical lesions. The double antibiotic paste (DAP), that excludes Minocycline, (which causes discoloration) ${ }^{19}$ has also been successfully used for regenerative endodontic therapy. The double antibiotic paste which contains Ciprofloxacin and Metranidazol was used for a period of 2 weeks. The mean period of time considered necessary for the bacterial disinfection of the canals, leaving the triple antibiotic paste in until they are clean, varies between 1 and 4 weeks, depending on the author. While Ding et al. ${ }^{8}$ (in their clinical study with humans) suggest waiting for 1 
week, some authors in experimental research, suggest 2 weeks ${ }^{20,21}$, others advocate leaving the triple antibiotic paste for 3 weeks ${ }^{22}$, and some have even proposed that it be left for four weeks ${ }^{9}$.

Inducing apical bleeding plays an important role in the success of this therapy. Using a file to irritate the apex, the fundamental reason is to cause the formation of a blood clot in the canal space, which then serves as a scaffold to enable the three-dimensional in-growth of new tissue ${ }^{10}$. Research shows that the inclusion of a blood clot in the root canal tends to improve the revascularization outcome, and that the induction of bleeding into the canal may provide stem cells that can induce dentin formation. Besides acting as a scaffold, the blood clot may also contain growth and differentiation factors that may be important for successful revascularization of the empty pulp canal. Ding et al suggested that failed regenerative procedures were attributed to the inability to evoke bleeding into the root canal. It is important to consider that if bleeding after canal disinfection is not achieved, clinicians should consider using an anesthetic without a vasoconstrictor when trying to induce bleeding because bleeding is easier when an anesthetic solution does not contain a vasoconstrictor. The bleeding should be allowed to reach a level of $3 \mathrm{~mm}$ below the cementoenamel junction, and the tooth is left for 15 minutes so that a blood clot forms. However, cases of successful revascularization using $\mathrm{Ca}(\mathrm{OH})_{2}$ without inducing bleeding have also been reported. This could be related to the presence of Stem cells from apical papilla (SCAP) in immature teeth, although induced bleeding will increase the chance of stem/progenitor cell migration.

Mineral trioxide aggregate is carefully placed over the blood clot, followed by a wet cotton pellet. A coronal seal with MTA is used because the material possesses an excellent sealing ability. To allow more root development, the coronal edge of the MTA should be placed 1-2 mm apical to the cementoenamel junction rather than 3-4 mm as described by Banchs and Trope. ${ }^{11}$

After 3 months follow up we observed that tooth was vital demonstrated by pulp vitality tests.

The technique referred to as regenerative endodontics, revascularization, or revitalization is therefore an effective and conservative option for necrotic immature teeth. In addition to promoting apical closure, it permits further root lengthening and thickening of dentinal walls, hence resolving the problem of susceptibility to fracture which was associated with apexification therapy. However, there are still too few large-scale clinical studies involving humans and more research is needed.

\section{Conclusion:-}

(1) For necrotic immature teeth, revascularization is a desirable alternative to apexification and shows both good short-term and long-term prognosis.

(2) A positive response to the pulp vitality test may appear after 3 months; this is an encouraging result, which indicates that this technique is preferable to apexification.

(3) For the future, more studies are needed to improve the results of regenerative endodontics therapy in immature teeth with pulp necrosis.

\section{References:-}

1. A. Mendoza-Mendoza, M. Biedma-Perea, A. Iglesias-Linares, C. Abalos-Labruzzi, and B. Solano-Mendoza, "Effect of mineral trioxide aggregate (MTA) pulpotomies in primary molars on their permanent tooth successors," American Journal of Dentistry, vol. 27, no. 5, pp. 268-272, 2014

2. D.-S. Kim, H.-J. Park, J.-H. Yeom et al., "Long-term follow-ups of revascularized immature necrotic teeth: three case reports," International Journal of Oral Science, vol. 4, no. 2, pp. 109-113, 2012.

3. R. Bose, P. Nummikoski, and K. Hargreaves, "A retrospectiveevaluation of radiographic outcomes in immature teeth with necrotic root canal systems treated with regenerative endodontic procedures," Journal of Endodontics, vol. 35, no. 10, pp. 1343- 1349, 2009.

4. A. Thomson and B. Kahler, "Regenerative endodontics - biologically-based treatment for immature permanent teeth: a case report and review of the literature," Australian Dental Journal, vol. 55, no. 4, pp. 446-452, 2010.

5. K. Reynolds, J. D. Johnson, and N. Cohenca, "Pulp revascularization of necrotic bilateral bicuspids using a modified novel technique to eliminate potential coronal discolouration: a case report," International Endodontic Journal, vol. 42, no. 1, pp. 84- 92, 2009.

6. M. Torabinejad, R. Corr, M. Buhrley, K. Wright, and S. Shabahang, "An animal model to study regenerative endodontics," Journal of Endodontics, vol. 37, no. 2, pp. 197-202, 2011. 
7. W. Zhang and P. C. Yelick, "Vital pulp therapy-current progress of dental pulp regeneration and revascularization,” International Journal of Dentistry, vol. 2010,Article ID 856087, 9 pages, 2010.

8. R. Y. Ding, G. S. Cheung, J. Chen, X. Z. Yin, Q. Q.Wang, and C. F. Zhang, "Pulp revascularization of immature teeth with apical periodontitis: a clinical study," Journal of Endodontics, vol. 35, no. 5, pp. 745-749, 2009.

9. M. Y.-H. Chen, K.-L. Chen, C.-A. Chen, F. Tayebaty, P. A. Rosenberg, and L. M. Lin, "Responses of immature permanent teeth with infected necrotic pulp tissue and apical periodontitis/abscess to revascularization procedures," International Endodontic Journal, vol. 45, no. 3, pp. 294-305, 2012.

10. G. T.-J. Huang, W. Sonoyama, Y. Liu, H. Liu, S. Wang, and S. Shi, "The hidden treasure in apical papilla: the potential role in pulp/dentin regeneration and bioroot engineering," Journal of Endodontics, vol. 34, no. 6, pp. 645-651, 2008.

11. F. Banchs and M. Trope, "Revascularization of immature permanent teeth with apical periodontitis: new treatment protocol?" Journal of Endodontics, vol. 30, no. 4, pp. 196-200, 2004.

12. M. C. Moreno-Hidalgo, C. Caleza-Jimenez, A. Mendoza-Mendoza, and A. Iglesias-Linares, "Revascularization of immature permanent teeth with apical periodontitis," International Endodontic Journal, vol. 47, no. 4, pp. 321-331, 2014.

13. R. Vijayaraghavan, V. M. Mathian, A. M. Sundaram, R. Karunakaran, and S. Vinodh, "Triple antibiotic paste in root canal therapy," Journal of Pharmacy And Bioallied Sciences, vol. 4, supplement 2, pp. 230-233, 2012.

14. A. L. Ritter, A. V. Ritter, V. Murrah, A. Sigurdsson, and M. Trope, "Pulp revascularization of replanted immature dog teeth after treatment with minocycline and doxycycline assessed by laser doppler flowmetry, radiography, and histology," Dental Traumatology, vol. 20, no. 2, pp. 75-84, 2004.

15. L. Spangberg, B. Engstrom, and K. Langeland, "Biologic effects of dental materials. 3. Toxicity and antimicrobial effect of endodontic antiseptics in vitro," Oral Surgery, Oral Medicine, Oral Pathology, vol. 36, no. 6, pp. 856-871, 1973.

16. J. C. Baumgartner and P. R. Cuenin, "Efficacy of several concentrations of sodium hypochlorite for root canal irrigation," Journal of Endodontics, vol. 18, no. 12, pp. 605-612, 1992.

17. R. M. Clarkson, A. J. Moule, and H. M. Podlich, "The shelf-life of sodium hypochlorite irrigating solutions," Australian Dental Journal, vol. 46, no. 4, pp. 269-276, 2001.

18. G. Jadhav, N. Shah, and A. Logani, "Revascularization with and without platelet-rich plasma in nonvital, immature, anterior teeth: a pilot clinical study," Journal of Endodontics, vol. 38, no. 12, pp. 1581-1587, 2012.

19. H. Park, B. Lee, Y. Hwang, I. Hwang, W. Oh, and H. Chang, "Treatment of non-vital immature teeth with amoxicillincontaining triple antibiotic paste resulting in apexification," Restorative Dentistry \& Endodontics, vol. 40, no. 4, p. 322, 2015.

20. L. A. B. da Silva, P. Nelson-Filho, R. A. B. da Silva et al., "Revascularization and periapical repair after endodontic treatment using apical negative pressure irrigation versus conventional irrigation plus triantibiotic intracanal dressing in dogs' teeth with apical periodontitis," Oral Surgery, Oral Medicine, Oral Pathology, Oral Radiology and Endodontology, vol. 109, no. 5, pp. 779-787, 2010.

21. W. Windley III, F. Teixeira, L. Levin, A. Sigurdsson, and M. Trope, "Disinfection of immature teeth with a triple antibiotic paste," Journal of Endodontics, vol. 31, no. 6, pp. 439-443, 2005.

22. S. Asgary, M. Fazlyab, and A.Nosrat, "Regenerative Endodontic Treatment versus Apical Plug in Immature Teeth: Three-Year Follow-Up,” Journal of Clinical Pediatric Dentistry, vol. 40, no. 5, pp. 356-360, 2016. 